\title{
Outsourcing Animal Drug Compounding
}

National Cancer Institute

\section{Source}

National Cancer Institute. Outsourcing Animal Drug Compounding. NCI Thesaurus. Code C122061.

Compounding drug dosage forms for animal consumption under a contract by other sources than the facilities that use the pharmaceutics. 